Abanico Veterinario. Enero-Diciembre 2021; 11:1-14. http://dx.doi.org/10.21929/abavet2021.40

Artículo Original. Recibido: 05/07/2021. Aceptado:08/10/2021. Publicado: 30/10/2021. Clave: e2021-53.

\title{
Características de conformación asociadas a producción y composición de la leche de vacas Holstein
}

\section{Conformation traits associated with milk yield and composition of Holstein cows}

\section{Cortes-Hernández José $^{\star 1}$ ID , Ruíz-López Felipe ${ }^{2}$ ID , García-Ruiz Adriana*^2 ID}

${ }^{1}$ Programa de Maestría y Doctorado en Ciencias de la Producción y de la Salud Animal, Universidad Nacional Autónoma de México. Ciudad Universitaria, Delegación Coyoacán, C.P 04510. Ciudad de México, México. ${ }^{2}$ Centro Nacional de Investigación Disciplinaria en Fisiología y Mejoramiento Animal, Instituto Nacional de Investigaciones Forestales Agrícolas y Pecuarias. Km. 1, Carretera a Colón, Col. Ajuchitlán Colón, C.P. 76280, Querétaro. México. "Autor responsable: Cortes-Hernández José. ${ }^{* *}$ Autor Correspondencia: García-Ruiz Adriana. E-mail: jgch1992@hotmail.com, ruiz.felipe@inifap.gob.mx, garcia.adriana@inifap.gob.mx

\section{RESUMEN}

Se evaluó la asociación entre los sistemas de conformación de tipo y la producción de leche (PL), grasa (PG) y proteína (PP) a través de la correlación entre las características de conformación (CC) y las predicciones de valores genéticos para leche (HTPL), grasa (HTPGK) y proteína (HTPPK) de ganado Holstein, para determinar en qué medida las CC influyen en la producción de los animales. El estudio incluyó 31,711 registros de CC, 87,871 de PL, 65,593 de PG y 43,717 de PP. Las CC se agruparon en cuatro sistemas generales: estructura y capacidad (SEC), patas y pezuñas (SP), ubre (SU) y anca (SA). También se evaluó el efecto de las clases de puntos finales (PF) en los niveles de PL, PG y PP a través de un análisis de varianza. La HTPL tuvo una correlación de 0.28 con SU, 0.17 con SA, 0.08 con SP y -0.05 con SEC $(\mathrm{P}<0.001)$. Las correlaciones de HTPGK y HTPPK con los diferentes sistemas fueron bajas, destacando las encontradas con $\mathrm{SU}, 0.15$ y 0.21 ( $P<0.001)$, respectivamente. Las asociaciones fenotípicas y las correlaciones de los valores genéticos reportadas en este estudio sugieren que la selección de vacas por CC puede modificar la producción de leche, grasa y proteína.

Palabras clave: Asociación fenotípica, Correlación entre valores genéticos, características de conformación, leche, grasa, proteína.

\begin{abstract}
The association between type conformation systems and milk (PL), fat (PG) and protein (PP) production was evaluated through the correlation between conformation traits (CC) and genetic value predictions for milk (HTPL), fat (HTPGK) and protein (HTPPK) of Holstein cattle, to determine the extent to which CC influence animal production. The study included 31,711 CC, 87,871 PL, 65,593 PG and 43,717 PP records. The CC were grouped into four general systems: structure and capacity (SEC), legs and hooves (SP), udder (SU) and haunch (SA). The effect of end point (FP) classes on PL, PG and PP levels was also evaluated through analysis of variance. HTPL had a correlation of 0.28 with SU, 0.17 with SA, 0.08 with SP and -0.05 with SEC $(P<0.001)$. The correlations of HTPGK and HTPPK with the different systems were low, highlighting those found with SU, 0.15 and $0.21(\mathrm{P}<0.001)$, respectively. The phenotypic associations and correlations of genetic values reported in this study suggest that selection of cows by CC can modify milk, fat and protein production.
\end{abstract}

Keywords: phenotypic association, correlation between genetic values, conformation traits, milk, fat, protein. 


\section{INTRODUCCIÓN}

El énfasis dado hasta la década de los 70's al mejoramiento genético para producción de leche en ganado Holstein causó un descenso en las tasas de progreso genético de características como la composición de la leche, aspectos reproductivos, de salud y de conformación, lo que generó repercusiones en la funcionalidad de los animales (Corrales et al., 2012; De Vries, 2017; Van Raden et al., 2021)

Los avances logrados en metodologías estadísticas aplicadas a la selección genética y el uso de herramientas computacionales, han permitido la inclusión de nuevas características en los programas de mejoramiento genético (Misztal \& Legarra, 2017). Algunos países han desarrollado metodologías sofisticadas para la inclusión de características con alto valor económico en los índices de selección, mientras que otros han incluido un gran número de características en sus evaluaciones, pero todos buscando a un animal más eficiente a través de la inclusión de caracteres que no sólo aumenten la cantidad de leche (Miglior et al., 2017), sino que reduzcan los costos de producción de la misma (Getu \& Misganaw, 2015).

Las características de conformación (CC) son rasgos morfológicos que determinan la aptitud funcional de los animales. Diversos estudios han demostrado su relación positiva con PL (Corrales et al., 2012; Manafiazar et al., 2015), sugiriendo que las CC pueden utilizarse como criterios de selección temprana para mejorar el desempeño de los animales e incrementar la producción, longevidad y la salud de los mismos (Battagin et al., 2013; Madrid \& Echeverri, 2014)

Desde los años 70 , en México se ha trabajado en la calificación de CC de ganado Holstein, basándose en el sistema recomendado por la Federación Mundial Holstein Friesian (World Holstein Friesian Federation o WHFF; Valencia et al., 2008). A partir de la evaluación de las CC, la información individual de producción, la composición de la leche y registros genealógicos se han predicho los valores genéticos de numerosas características que han permitido identificar los animales más productivos (Getu \& Misganaw, 2015), funcionales por sus CC y adaptados a sus necesidades (Zavadilová \& Štt́pková, 2012). El objetivo del presente estudio fue evaluar la asociación entre los sistemas de conformación y las clases de puntos finales (PF), con la producción de leche $(\mathrm{PL})$, grasa $(\mathrm{PG})$ y proteína $(\mathrm{PP})$ a través de la correlación de Pearson entre las mediciones directas (fenotipos) y las predicciones de valor genético de ganado Holstein de México.

\section{MATERIAL Y MÉTODOS}

El estudio incluyó información de animales Holstein nacidos de 1992 a 2010, que estuvieron en el sistema de control de producción y en el programa de calificación de conformación de la Asociación Holstein de México (AHM). La información fue recolectada en 94 hatos ubicados en los estados de la República Mexicana: Aguascalientes, Baja California Norte, Chihuahua, Coahuila, Estado de México, Guanajuato, Jalisco, 
Querétaro, Michoacán, Nayarit, Puebla, San Luis Potosí, Tlaxcala y Zacatecas. Se incluyeron registros fenotípicos de 87,871 lactaciones para producción de leche y 65,593 lactaciones para componentes de la leche (grasa y proteína) correspondientes a 43,717 y 31,711 animales, respectivamente. Las producciones acumuladas de leche y sus componentes se obtuvieron como la suma de las producciones de las tres primeras lactaciones ajustadas a 305 días y equivalente de madurez (Toledo et al., 2014). Para evitar sesgos por edades de los animales, solo se incluyeron animales que tuvieron oportunidad de alcanzar la tercera lactación. También, se incluyó información fenotípica y de predictores de valores genéticos de las siguientes CC: estatura (STA), altura a la cruz (ACRZ), angulosidad (AG), tamaño (TAMÑ), profundidad corporal (PROFD), fortaleza del lomo (LOM), anchura de pecho (ANPE), punta del anca (PUNA), anchura del anca (ANCA), calidad de hueso (CALHU), vista lateral de las patas traseras (VLPT), vista posterior de las patas traseras (VPPT), ángulo de la pezuña (ANPEZ), profundidad del talón (PROTL), posición de pezones posteriores (POSPP), posición de pezones anteriores (POSPA), longitud de los pezones (LONPE), profundidad de la ubre (PU), inserción de la ubre anterior (IAU), altura de inserción de la ubre posterior (AIUP), ligamento medio suspensorio (LM), textura de la ubre (TEX), anchura de inserción de la ubre posterior (ANIUP) y puntos finales (PF). La descripción de cada una de las anteriores características se presenta en la Tabla 1, considerando que solo algunas de ellas tienen una calificación objetiva, con diferencias en centímetros y en otras la calificación es subjetiva, por lo que el calificador debe ser certificado por organismos internacionales de la raza.

Las CC fueron evaluadas en una escala del 1 al 9 con excepción de PF que fue evaluada en una escala de 70 a 89 puntos. Las CC se agruparon en cuatro sistemas de conformación ponderándose las características que formaron parte de cada uno de los sistemas por los pesos que recomienda la WHFF (Madrid \& Echeverri, 2014; De Jong, 2020) y que se utilizan en la AHM. Finalmente se evaluó el impacto de los sistemas sobre PL, PG y PP. Los sistemas estudiados fueron:

$$
\begin{gathered}
\text { Sistema de ubre (SU) } \\
S U=(P U * 0.16)+(T E X * 0.14)+(L M * 0.14)+(I A U * 0.18)+(P O S P A * 0.07) \\
+(L O N P E * 0.02)+(A I U P * 0.12)+(A I U P * 0.10)+(P O S P P * 0.07) \\
\text { Sistema de patas y pezuñas (SP) } \\
S P=(A N P E Z * 0.18)+(P R O T L * 0.22)+(C A L H U * 0.12)+(V L P T * 0.17)+(V P P T \\
* 0.31) \quad \text { Sistema de estructura y capacidad (SEC) } \\
S E C=(S T A * 0.12)+(A C R Z * 0.03)+(T A M \tilde{N} * 0.17)+(A N P E * 0.23)+(P R O F D * 0.17) \\
+(L O M * 0.28) \quad \text { Sistema del anca (SA) } \\
S A=(P U N A * 0.62)+(A N C A * 0.38) \\
3
\end{gathered}
$$


Tabla 1 Características de conformación evaluadas

\begin{tabular}{|c|c|}
\hline Característica & Puntos anatómicos de referencia para su calificación \\
\hline Tamaño (TAMÑ) & $\begin{array}{l}\text { Agrupa características como altura a la cruz, altura al anca, } \\
\text { angulosidad, profundidad corporal, ancho de pecho y fortaleza } \\
\text { del lomo. }\end{array}$ \\
\hline Textura de la ubre (TEX) & $\begin{array}{l}\text { Apariencia suave, plegable, carnosa, con basta irrigación y de } \\
\text { buena conformación. }\end{array}$ \\
\hline $\begin{array}{l}\text { 3- } \quad \text { Posición de pezones } \\
\text { posteriores (POSPP) }\end{array}$ & $\begin{array}{l}\text { Ubicación de los pezones posteriores con respecto al centro del } \\
\text { cuarto. }\end{array}$ \\
\hline $\begin{array}{l}\text { 4- Longitud de los pezones } \\
\text { (LONPE) }\end{array}$ & $\begin{array}{l}\text { Calificación de pezones anteriores, } 1 \text { centímetro equivale a un } \\
\text { punto, escala de } 1 \text { a } 9 \mathrm{~cm} \text {. }\end{array}$ \\
\hline $\begin{array}{l}\text { 5- Posición de pezones } \\
\text { anteriores (POSPA) }\end{array}$ & $\begin{array}{l}\text { Mide la posición que ocupan los pezones anteriores respecto al } \\
\text { eje central de los cuartos. }\end{array}$ \\
\hline $\begin{array}{l}\text { 6- } \quad \text { Ligamento medio } \\
\text { suspensorio (LM) }\end{array}$ & Profundidad del surco en la base posterior de la ubre. \\
\hline 7- $\quad$ Profundidad de la ubre (PU) & $\begin{array}{l}\text { Distancia entre la parte más baja del piso de la ubre y la altura de } \\
\text { los corvejones. Cada } 3 \mathrm{~cm} \text { de diferencia representa un punto en } \\
\text { la escala de medición de la característica, tomando como } \\
\text { referencia el nivel a los corvejones cuya calificación es } 3 \text {. }\end{array}$ \\
\hline $\begin{array}{l}\text { 8- } \quad \text { Altura de inserción de la ubre } \\
\text { posterior (AIUP) }\end{array}$ & $\begin{array}{l}\text { Distancia entre la vulva y el inicio de la ubre. Este valor se } \\
\text { relaciona con la estatura del animal. }\end{array}$ \\
\hline $\begin{array}{l}\text { 9- Inserción de ubre anterior } \\
(\mathrm{IAU})\end{array}$ & $\begin{array}{l}\text { Ubicación y la fuerza con que la ubre se adhiere a la pared } \\
\text { abdominal. }\end{array}$ \\
\hline 10- $\quad$ Angulo de pesuña (ANPEZ) & $\begin{array}{l}\text { Ángulo formado entre la parte anterior de la pezuña y el piso del } \\
\text { miembro torácico derecho. }\end{array}$ \\
\hline $\begin{array}{l}\text { 11- Vista lateral de patas } \\
\text { traseras (VLPT) }\end{array}$ & Ángulo formado en la parte delantera de los corvejones. \\
\hline $\begin{array}{l}\text { 12- Vista posterior de patas } \\
\text { traseras (VPPT) }\end{array}$ & $\begin{array}{l}\text { Dirección que adoptan los miembros posteriores vistos desde } \\
\text { atrás. }\end{array}$ \\
\hline 13- Anchura del anca (ANCA) & $\begin{array}{l}\text { Distancia entre la punta de los huesos posteriores de la cadera } \\
\text { (isquiones) } 2 \mathrm{~cm} \text { por punto. }\end{array}$ \\
\hline 14- $\quad$ Punta del anca (PUNA) & Diferencia de la medición de la altura entre íleon e isquion. \\
\hline 15- $\quad$ Fortaleza del lomo (LOM) & $\begin{array}{l}\text { Debe ser recto con una ligera inclinación hacia atrás y de una } \\
\text { apariencia firme. }\end{array}$ \\
\hline 16- $\quad$ Altura a la cruz (ACRZ) & $\begin{array}{l}\text { Medida exacta que va desde el suelo en el miembro anterior a la } \\
\text { cruz del animal; } 1.35 \mathrm{~m} \text { a } 1.60 \mathrm{~m}, 3 \text { puntos por } \mathrm{cm} \text {. }\end{array}$ \\
\hline $\begin{array}{l}\text { 17- Profundidad corporal } \\
\text { (PROFD) }\end{array}$ & $\begin{array}{l}\text { Distancia entre la columna vertebral y el ombligo al nivel de la } \\
\text { última costilla, su punto de referencia es óptico. }\end{array}$ \\
\hline 18- $\quad$ Angulosidad (AG) & $\begin{array}{l}\text { Separación y ángulo de las costillas. La evaluación se hace con } \\
\text { base en tres componentes, ángulo y apertura de costilla ( } 80 \%) \text {, } \\
\text { calidad del hueso (20\%). }\end{array}$ \\
\hline 19- $\quad$ Anchura del pecho (ANPE) & $\begin{array}{l}\text { Medida entre las patas delanteras en su parte más alta. Se usa } \\
\text { una escala de referencia de } 13 \text { a } 29 \text { centímetros, } 2 \mathrm{~cm} \text { por punto. }\end{array}$ \\
\hline 20- Estatura (STA) & $\begin{array}{l}\text { Medida exacta que va desde el suelo a la grupa del animal escala; } \\
1.30 \mathrm{~m} \text { a } 1.54 \mathrm{~m}, 3 \text { puntos por } \mathrm{cm} \text {. }\end{array}$ \\
\hline 21- $\quad$ Calidad del hueso (CALHU) & $\begin{array}{l}\text { Apariencia de huesos planos no muy gruesos que den al animal } \\
\text { una apreciación fenotípica de feminidad }\end{array}$ \\
\hline $\begin{array}{l}\text { 22- Ancho de inserción de la } \\
\text { ubre posterior (ANIUP) }\end{array}$ & $\begin{array}{l}\text { Mide la anchura que tiene la ubre cerca de la altura de inserción } \\
\text { posterior }\end{array}$ \\
\hline $\begin{array}{l}\text { 23- } \text { Profundidad del talón } \\
\text { (PROTL) }\end{array}$ & $\begin{array}{l}\text { Mide la distancia que hay del talón al piso esto con base al ángulo } \\
\text { de la pezuña, } 45^{\circ} \text { normalmente. }\end{array}$ \\
\hline 24- $\quad$ Puntos finales & $\begin{array}{l}\text { Calificación general del animal, ponderada en función de las } \\
\text { calificaciones obtenidas por sistema. Tiene una escala del } 79 \text { a } \\
89 \text {. }\end{array}$ \\
\hline
\end{tabular}


Para estudiar el efecto de las CC en las características de producción, se generaron cuatro clases fenotípicas, tomando como referencia el valor de PF obtenido en la evaluación de conformación realizada en la primera lactación de cada animal, siguiendo las recomendaciones de la WHFF, (2018). Las clases fueron: regular (RE) con 74 puntos o menos, buena (BU) entre 75 y 79 puntos, más buena (MB) entre 80 y 84 puntos y muy buena (MU) con 85 puntos o más. Para evaluar el efecto de las clases por CC en la permanencia de los animales en el establo, se analizó el promedio del número de lactaciones por animal. El efecto de la conformación evaluada como clase de PF en PL, PG, PP y el número de lactaciones por animal (NLAC), se evaluó a través de un análisis de varianza midiendo las diferencias entre las medias de cuadrados mínimos con del procedimiento GLM usando el paquete estadístico SAS 9.3 (SAS Institute. 2019).

Los valores genéticos predichos usados en el estudio para las diferentes características, fueron obtenidos y proporcionados por el INIFAP y la AHM (Ruíz et al., 2020), institución encargada de realizar las evaluaciones de la raza Holstein. Para determinar la asociación fenotípica de las CC con PL, PG y PP, así como la asociación entre sus valores genéticos predichos (que en el caso de las características de producción son las habilidades de transmisión predicha para PL, PG y PP; HTPL, HTPGK y HTPPK respectivamente) se calcularon los coeficientes de correlación de Pearson (Wayne, 2017), usando el programa SAS Institute (2019).

\section{RESULTADOS Y DISCUSIÓN}

\section{Asociaciones fenotípicas}

Los resultados obtenidos mostraron que más del $60 \%$ de los animales incluidos en el estudio cuentan con una calificación igual o mayor que $80 \mathrm{PF}$, lo que indica que más de la mitad de la población se encuentra en las dos clases de conformación superiores (MB y MU). Tabla 2 muestra que existe diferencia estadística $(P<0.001)$ entre las clases de PF sobre PL, PG, PP y NLAC, observándose que al aumentar la clase de PF se incrementa el nivel de producción en las tres características estudiadas (PL, PG y PP) y el NLAC.

Los animales MU tuvieron 0.26 lactaciones más que los animales RE. Esta diferencia representa aproximadamente 79 días más en lactación, tiempo que permite incrementar la producción total de los animales y hace más rentable cada animal. Estos resultados se ven reflejados en los promedios de $\mathrm{PL}$ de los animales de clase $\mathrm{MU}$, que presentaron $21 \%$ más PL que los de la clase RE. Los resultados obtenidos en la población bajo estudio, coinciden con los reportados en estudios previos realizados en ganado Holstein en Colombia, dónde mostraron que las CC están correlacionadas positivamente con la producción de leche Madrid \& Echeverri, 2014. Las CC a diferencia de los rasgos productivos, pueden ser herramientas de selección temprana en ganado lechero, ya que la calificación puede ser obtenida al iniciar la primera lactación; además, se ha reportado 
que estos caracteres presentan correlaciones genéticas positivas con rasgos de interés económico, como por ejemplo en producción de leche, la composición de la misma y salud de la ubre (Duru et al., 2012).

Tabla 2. Medias mínimo cuadráticas y errores estándar para producción de leche, producción de grasa y producción de proteína de acuerdo a su clasificación de puntos finales

\begin{tabular}{ccccccc}
\hline $\begin{array}{c}\text { Clase de } \\
\text { puntos } \\
\text { finales }\end{array}$ & $\begin{array}{c}\text { Registros } \\
\text { de leche }\end{array}$ & Lactaciones & $\begin{array}{c}\text { Producción } \\
\text { de leche }\end{array}$ & $\begin{array}{c}\text { Registros de } \\
\text { grasa y proteína }\end{array}$ & $\begin{array}{c}\text { Producción } \\
\text { de grasa }\end{array}$ & $\begin{array}{c}\text { Producción de } \\
\text { proteína }\end{array}$ \\
\hline RE & 1,762 & $2.02 \pm 0.02^{\mathrm{a}}$ & $21,231 \pm 253^{\mathrm{a}}$ & 1,237 & $674 \pm 10.8^{\mathrm{a}}$ & $548 \pm 10.7^{\mathrm{a}}$ \\
$\mathrm{BU}$ & 14,658 & $2.19 \pm 0.01^{\mathrm{b}}$ & $23,509 \pm 88^{\mathrm{b}}$ & 10,491 & $751 \pm 3.7^{\mathrm{b}}$ & $625 \pm 3.7^{\mathrm{b}}$ \\
MB & 23,329 & $2.22 \pm 0.01^{\mathrm{c}}$ & $24,180 \pm 69^{\mathrm{c}}$ & 17,206 & $776 \pm 2.9^{\mathrm{c}}$ & $664 \pm 2.9^{\mathrm{c}}$ \\
MU & 3,968 & $2.28 \pm 0.01^{\mathrm{d}}$ & $25,804 \pm 168^{\mathrm{d}}$ & 2,777 & $827 \pm 7.2^{\mathrm{d}}$ & $729 \pm 7.1^{\mathrm{c}}$ \\
\hline
\end{tabular}

RE: Regular, BU: Buena, MB: Más buena, MU: Muy buena.

Superíndices diferentes en la misma columna indican una diferencia estadística significativa $(P<0.001)$.

Al igual que para leche, para componentes (PG y PP), las vacas con la clase más alta (MU) tuvieron producción mayor (827 y $729 \mathrm{Kg}$, respectivamente) aunque en el caso de PP no hubo diferencia significativa $(P<0.001)$ entre vacas de las clases MB y MU. Los resultados mostraron que los animales de la clase más baja (RE) tuvieron $22 \%$ menor PG y $33 \%$ menor PP en comparación con los animales de la clase más alta (MU). Estos resultados corroboran la relación positiva que existe entre producción de leche y componentes con la clasificación de CC (Madrid \& Echeverri, 2014), y corroboran los reportes de correlaciones genéticas positivas (Zavadilová \& Štt́pková, 2012) que indican que las vacas con mejor estructura anatómica, tienden a mejorar su eficiencia fisiológica. Las correlaciones entre los sistemas de conformación fueron de moderadas a bajas, destacando la que existe entre SU y SEC (0.40), entre SU y SP (0.29) y, entre SEC y SA (0.27) (Tabla 3).

La magnitud de estas correlaciones muestra la dependencia anatómica y funcional que existe entre los sistemas y la posibilidad de ser mejorados por selección de manera individual; ya que, por ejemplo, la VPPT Y VLPT están relacionadas con la inclinación de anca y el desarrollo y tamaño de la ubre están altamente influenciados por el TAMÑ y STA, características del SEC. Las correlaciones entre los sistemas de las CC y las características productivas fueron generalmente bajas, solo destacaron las correlaciones del SP, que presentó una correlación de 0.47 con PP y de 0.38 con PG. No se han reportado resultados similares en otras poblaciones. 
Tabla 3. Correlaciones fenotípicas (debajo de la diagonal) y de valores genéticos (arriba de la diagonal) entre los sistemas de conformación con producción de leche, grasa y proteína

\begin{tabular}{|c|c|c|c|c|c|c|c|}
\hline & \multicolumn{4}{|c|}{ Sistema } & \multicolumn{3}{|c|}{ Producción } \\
\hline & Ubre & $\begin{array}{l}\text { Patas y } \\
\text { Pesuñas }\end{array}$ & $\begin{array}{c}\text { Estructura y } \\
\text { Capacidad }\end{array}$ & Anca & Leche & Grasa & Proteína \\
\hline Ubre & & $0.53^{*}$ & $0.45^{*}$ & $0.13^{*}$ & $0.28^{*}$ & $0.15^{\star}$ & $0.21^{*}$ \\
\hline Patas y Pezuñas & $0.29^{*}$ & & $0.47^{\star}$ & $0.08^{*}$ & $0.08^{*}$ & $0.06^{*}$ & $0.08^{*}$ \\
\hline Estructura y Capacidad & $0.40^{*}$ & $0.18^{*}$ & & $0.30^{*}$ & $-0.05^{*}$ & -0.01 & -0.08 \\
\hline Anca & $0.08^{*}$ & -0.03 & $0.27^{*}$ & & $0.17^{\star}$ & $0.08^{*}$ & $0.09^{*}$ \\
\hline Leche & $0.07^{\star}$ & $0.16^{*}$ & 0.02 & 0 & & $0.69^{*}$ & $0.76^{\star}$ \\
\hline Grasa & $0.11^{*}$ & $0.38^{*}$ & 0.01 & $-0.05^{\star}$ & $0.64^{*}$ & & $0.92^{*}$ \\
\hline Proteína & $0.11^{*}$ & $0.47^{\star}$ & 0.02 & $-0.05^{\star}$ & $0.63^{*}$ & $0.95^{\star}$ & \\
\hline
\end{tabular}

${ }^{*}$ Correlaciones estadísticamente significativas $(\mathrm{P}<0.001)$

Correlaciones entre valores genéticos

La correlación encontrada entre HTPL y HTPPK fue alta y positiva (0.76), parecida a la encontrada con HTPGK (0.69), lo que significa que vacas con mayor valor genético predicho para producción láctea tienden a poseer un mayor valor genético para la producción de sólidos totales (Madrid \& Echeverri, 2014). Las correlaciones encontradas entre los sistemas de conformación con HTPGK y HTPPK fueron en su mayoría positivas. EI SEC fue el que mostró correlaciones más bajas con HTPL $(-0.05)$, y sin significancia para HTPGK y HTPPK. La correlación entre SU y HTPL fue baja y positiva (0.28), siendo éste el sistema que más se correlaciona con HTPL.

De todas las CC del SEC, la correlación encontrada entre STA y HTPL fue la que presentó mayor valor (0.21) (Tabla 4).

Tabla 4. Estimadores de los coeficientes de correlación de Pearson entre características de conformación de cuerpo y anca con los predictores de valores genéticos para producción de leche, grasa y proteína

\begin{tabular}{lcccccccc}
\hline Estatura & $\begin{array}{c}\text { Altura a } \\
\text { la cruz }\end{array}$ & Tamaño & $\begin{array}{c}\text { Ancho del } \\
\text { pecho }\end{array}$ & Profundidad & Lomo & $\begin{array}{c}\text { Punta } \\
\text { del anca }\end{array}$ & $\begin{array}{c}\text { Ancho } \\
\text { del anca }\end{array}$ \\
\hline HTPL & $0.21^{*}$ & $-0.07^{*}$ & 0.01 & $-0.06^{*}$ & $-0.12^{*}$ & 0.02 & $0.08^{*}$ & $0.18^{*}$ \\
HTPGK & $0.16^{*}$ & $-0.04^{*}$ & $0.08^{*}$ & 0.01 & $-0.05^{*}$ & -0.00 & $0.04^{*}$ & $0.09^{*}$ \\
HTPPK & $0.17^{*}$ & $-0.07^{*}$ & $0.05^{*}$ & $-0.06^{*}$ & $-0.12^{*}$ & $-0.05^{*}$ & $0.07^{*}$ & $0.08^{*}$ \\
\hline
\end{tabular}

HTPL: Predicción de valor genético para leche, HTPGK: Predicción de valor genético para grasa, HTPPK: Predicción de valor genético para proteína.

${ }^{*}$ Correlaciones estadísticamente significativas $(\mathrm{P}<0.001)$.

A pesar de que las estimadas en este estudio fueron correlaciones entre valores genéticos, fueron similares a las correlaciones genéticas reportadas en una población de vacas Holstein primíparas de Turquía por Tapki \& Ziya GÜZEY (2013), que fue de 0.24 
pero mayor que la reportada en otra población de la misma raza en vacas de tercer parto en el mismo país que fue de 0.14 (Duru et al., 2012), lo que indica que la estatura del animal influye en la capacidad de producción de leche. En los establos, se recomienda seleccionar a los animales que tengan una calificación de 7 para STA, ya que estás vacas tienen la misma capacidad de producir leche que vacas de mayor tamaño por lo que se reduce el costo energético de mantenimiento en estos animales. La correlación encontrada entre PUNA y HTPL fue baja y positiva (0.08), difiriendo parcialmente con otros autores (Weller \& Ezra, 2016) quienes reportaron valores negativos y bajos $(-0.04)$. La correlación entre ANCA y HTPL fue mayor (0.18) y similar a los reportados en ganado Holstein de Turquía (Duru et al., 2012) donde reportaron una correlación de 0.19 entre las mismas características. El ANCA determina la separación entre las patas posteriores de la vaca que entre más separadas estén, más amplia será la ubre posterior, permitiendo que esta almacene y produzca una mayor cantidad de leche (Getu \& Misganaw, 2015). Las correlaciones entre ANCA con HTPGK y HTPPK fueron bajas y significativamente diferentes $p<0.001$ ( 0.09 y 0.08 , respectivamente).

EI SU tuvo una correlación positiva con HTPL (0.28), resultado que indica que vacas con ubres fuertes, grandes y bien implantadas producen mayor cantidad de leche. De las características del SU, la PU tuvo una correlación baja y negativa con HTPL (-0.05), (Tabla 5).

Tabla 5. Estimadores de los coeficientes de correlación de Pearson entre características de conformación de ubre con los predictores de valores genéticos para producción de leche, grasa y proteína

\begin{tabular}{|c|c|c|c|c|c|c|c|c|c|}
\hline & \multirow{2}{*}{$\begin{array}{l}\text { Profundidad } \\
\text { de ubre }\end{array}$} & \multirow{2}{*}{ Textura } & \multirow{2}{*}{$\begin{array}{l}\text { Ligamento } \\
\text { medio }\end{array}$} & \multirow{2}{*}{$\begin{array}{l}\text { Inserción } \\
\text { anterior } \\
\text { de ubre }\end{array}$} & \multirow{2}{*}{$\begin{array}{l}\text { Posición de } \\
\text { pezones } \\
\text { anteriores }\end{array}$} & \multirow{2}{*}{$\begin{array}{l}\text { Longitud de } \\
\text { pezones }\end{array}$} & \multicolumn{2}{|c|}{$\begin{array}{l}\text { Inserción de ubre } \\
\text { posterior }\end{array}$} & \multirow{2}{*}{$\begin{array}{c}\text { Posición de } \\
\text { pezones } \\
\text { posteriores }\end{array}$} \\
\hline & & & & & & & Altura & Anchura & \\
\hline HTPL & $-0.05^{\star}$ & $0.33^{*}$ & $0.40^{*}$ & $0.26^{\star}$ & $0.35^{\star}$ & $0.05^{\star}$ & $0.33^{*}$ & $0.37^{\star}$ & $0.47^{*}$ \\
\hline HTPGK & 0.01 & $0.24^{*}$ & $0.29^{*}$ & $0.21^{*}$ & $0.24^{*}$ & $-0.05^{\star}$ & $0.24^{*}$ & $0.24^{*}$ & $0.31^{*}$ \\
\hline HTPPK & $0.02^{*}$ & $0.27^{*}$ & $0.34^{*}$ & $0.20^{*}$ & $0.29^{*}$ & $-0.07^{*}$ & $0.30^{*}$ & $0.31^{*}$ & $0.35^{\star}$ \\
\hline
\end{tabular}

HTPL: Predicción de valor genético para leche, HTPGK: Predicción de valor genético para grasa, HTPPK: Predicción de valor genético para proteína.

*Correlaciones estadísticamente significativas $(\mathrm{P}<0.001)$.

Para las mismas características, en bovinos Holstein de Italia (Samoré et al., 2010) reportaron correlaciones genéticas negativas $(-0.37)$, pero menores a los reportados en Colombia (Corrales et al., 2012) dónde encontraron una correlación negativa y alta $(-0.72)$. Los resultados muestran que, en esas poblaciones, las vacas con alto potencial de producción láctea tienen una ubre con menor profundidad, ya que los animales con ubres poco profundas tienen poca cantidad de tejido mamario y por lo tanto su capacidad de producción y almacenamiento es limitada (Piccardi et al., 2012). 
Similarmente a PU, LONPE presentó una correlación de valores genéticos baja con HTPL (0.05 respectivamente; $p<0.001)$. El resto de las características del SU mostraron una correlación positiva y moderada con la HTPL, destacando la existente entre HTPL con POSPP (0.47) y LM (0.40) (Tabla 5). Estos resultados coinciden con los reportados en Colombia (Corrales et al., 2012), población en la que destacó una correlación positiva entre HTPL con AIUP y LM, se presenta porque ubres anchas y con inserción alta se relacionan con cantidad de tejido mamario y mayor capacidad de almacenamiento de la leche. La relación entre HTPL y POSPP puede deberse en gran parte a que los animales que tienen bien colocados los pezones, tienen mayor facilidad a la ordeña, por lo tanto, tienden a producir mayor cantidad de leche y al mismo tiempo pueden presentar menor incidencia de enfermedades de la glándula mamaria. Las correlaciones entre las características del SU y HTPGK y HTPPK presentaron tendencias similares a las de HTPL, resultados que indican que la buena conformación de la ubre contribuye a aumentar la capacidad de producción de leche, grasa y proteína (Ptak et al., 2011).

Diversos autores coinciden en que las vacas con buena locomoción tienden a ser altas productoras de leche y permanecer en el hato por más tiempo que las vacas con malas puntaciones para el sistema de patas (Wasana et al., 2015). En este estudio, las correlaciones de valores genéticos entre SP y HTPGK, y HTPPK fueron bajas (0.06 y 0.08 respectivamente), similares a las correlaciones genéticas reportadas en una población Holstein de Italia (Battagin et al., 2013), quienes encontraron una correlación de 0.07 con HTPGK y de 0.02 con HTPPK. La correlación del SP con HTPL también fue baja (0.08), diferente a lo reportado en la misma población, en la cual estimaron una correlación de 0.24 .

Las características individuales del SP que presentaron mayor correlación con HTPL fueron ANPEZ y CALHU ( 0.22 y 0.34 , respectivamente), mientras que estas mismas CC presentaron una correlación media con HTPGK y HTPPK (Tabla 6).

En ganado Holstein de España, Pérez-Cabal \& Alenda (2006) reportaron una correlación genética positiva y baja de 0.12 entre VPPT y HTPL, resultados que coinciden con los reportados en este estudio, en el que se encontró una correlación de 0.16. En bovinos Holstein del Reino Unido Ptak et al. (2011) sugirieron que las patas y pezuñas se relacionan de manera indirecta con la producción de leche, ya que vacas con malas puntuaciones para ANPEZ, VLPT y VPPT muestran un deterioro en longevidad, producción y fertilidad, además de ser características relacionadas con la repetición de servicio a los 56 días; sugiriendo que los animales con buena puntuación para patas podrían tener menos probabilidad de repetición de servicio, reduciendo días abiertos (Wall et al., 2005). La importancia de las características del SP en los sistemas de producción, radica en que las vacas, diariamente tienen que desplazarse para poder ser ordeñadas y si no tienen buena fortaleza y locomoción en los miembros, al paso del tiempo puede causar un deterioro en la vida productiva (Kern et al., 2015). 
Tabla 6. Estimadores de los coeficientes de correlación de Pearson entre características de conformación de patas y angulosidad con los predictores de valores genéticos para producción de leche, grasa y proteína

\begin{tabular}{|c|c|c|c|c|c|c|}
\hline & $\begin{array}{c}\text { Angulo de } \\
\text { pezuña }\end{array}$ & $\begin{array}{c}\text { Profundidad } \\
\text { de talón }\end{array}$ & $\begin{array}{c}\text { Calidad } \\
\text { de hueso }\end{array}$ & $\begin{array}{l}\text { Vista lateral de } \\
\text { patas traseras }\end{array}$ & $\begin{array}{c}\text { Vista posterior de } \\
\text { patas traseras }\end{array}$ & Angulosidad \\
\hline HTPL & $0.22^{*}$ & $0.16^{*}$ & $0.34^{*}$ & $0.12^{*}$ & $0.16^{\star}$ & $0.34^{*}$ \\
\hline HTPGK & $0.19^{*}$ & $0.17^{*}$ & $0.24^{*}$ & $0.06^{*}$ & $0.15^{\star}$ & $0.27^{*}$ \\
\hline HTPPK & $0.20^{*}$ & $0.17^{*}$ & $0.28^{*}$ & $0.08^{*}$ & $0.16^{*}$ & $0.30^{*}$ \\
\hline
\end{tabular}

HTPL: Predicción de valor genético para leche, HTPGK: Predicción de valor genético para grasa, HTPPK: Predicción de valor genético para proteína.

${ }^{*}$ Correlaciones estadísticamente significativas $(\mathrm{P}<0.001)$.

La correlación entre AG y HTPL encontrada en este estudio fue positiva (0.34) (Tabla 6), similar a la encontrada en la población Holstein de Brasil (Campos et al., 2015) donde reportaron una correlación (0.38). De la misma manera, en vacas Holstein de Turquía, Duru et al. (2012) reportaron una correlación positiva de 0.21 para las mismas características, mientras que en Colombia, Corrales et al. (2012) reportaron una correlación más baja (0.14). Aunque la metodología para encontrar relación entre características fue diferente, los resultados en México son similares a los presentados en Italia en ganado de la raza Suizo Pardo (Samoré et al., 2010), con una correlación entre AG y HTPL de 0.36. Con los resultados encontrados se puede inferir que las vacas más angulosas, tienden a producir más leche, debido a que animales con mayor separación entre costillas y mayor ángulo, tienen una mejor capacidad corporal; además los animales tienden a presentar huesos más planos, aspecto importante en la deposición y extracción de calcio durante la lactación (Carvajal-Hernández et al., 2002).

\section{CONCLUSIONES E IMPLICACIONES}

Los resultados muestran que es posible utilizar datos fenotípicos de las características de conformación como predictores tempranos de la producción láctea del ganado Holstein, destacando las características del SU y SP; pero es recomendable tener en cuenta las demás características morfológicas. La obtención de valores genéticos y realizar programas de mejoramiento permitirá contar con vacas más funcionales y rentables. La cantidad de proteína y grasa también están asociadas positivamente con algunas características de conformación, especialmente del sistema de ubre, como por ejemplo POSPP, LM y ANIUP. Para determinar la relación genética entre las CC con las características productivas, es necesario realizar análisis bivariados y determinar las correlaciones genéticas entre las diferentes características, aspecto que puede reforzar los resultados encontrados en este estudio. 


\section{AGRADECIMIENTOS Y FUENTE FINANCIADORA}

Agradecimiento la Asociación Holstein de México, por la información proporcionada.

Este Proyecto fue financiado por el Centro Nacional de Investigación Disciplinaria en Fisiología y Mejoramiento Animal del Instituto Nacional de Investigaciones Forestales Agrícolas y Pecuarias, con el nombre "Estudio de la consanguinidad y su efecto sobre características productivas y reproductivas en ganado Holstein" con número SIGI: 11513634465.

\section{LITERATURA CITADA}

ASOCIACIÓN HOLSTEIN DE MÉXICO. 2021. Holstein de México, A.C. Sitio web: https://holstein.com.mx/

BATTAGIN M, Sartori C, Biffani S, Penasa M, Cassandro M. 2013. Genetic parameters for body condition score, locomotion, angularity, and production traits in Italian Holstein cattle. Journal of Dairy Science. 96(8):5344-5351. ISSN 0022-0302. https://doi.org/10.3168/jds.2012-6352

CAMPOS RV, Cobuci JA, Kern EL, Costa CN, McManus CM. 2015. Genetic parameters for linear type traits and milk, fat, and protein production in holstein cows in Brazil. AsianAustralas J Anim Sci. 28(4):476-84. PMID: 25656190; PMCID: PMC4341096. https://doi.org/10.5713/ajas.14.0288

CARVAJAL-HERNÁNDEZ M, Valencia-Heredia RE, Segura-Correa CJ. 2002. Duración de la lactancia y producción de leche de vacas Holstein en el Estado de Yucatán, México. Revista Biomédica. 13(1):25-31. https://doi.org/10.32776/revbiomed.v13i1.292

CORRALES J, Cerón-Muñoz M, Cañas J, Herrera C, Calvo S. 2012. Parámetros genéticos de características de tipo y producción en ganado Holstein del departamento de Antioquia. MVZ Córdoba. 17(1):2870-2877. https://www.imbiomed.com.mx/articulo.php?id=83599

DE JONG G. 2020. Progress of type armonisation. v1. http://www.whff.info/documentation/documents/WHFFprogressoftypeharmonisation2020 .pdf

DE VRIES A. 2017. Economic trade-offs between genetic improvement and longevity in dairy cattle. PMID: 28215896. Journal of Dairy Science. 100(5):4184-4192. https://doi.org/10.3168/jds.2016-11847 
DURU S, Kumlu S, Tuncel E. 2012. Estimation of variance components and genetic parameters for type traits and milk yield in Holstein cattle. Turkish Journal of Veterinary and Animal Sciences. 36(6):585-591. https://doi.org/10.3906/vet-1012-660

GETU A. Misganaw G. 2015. The Role of Conformational Traits on Dairy Cattle Production and Their Longevities. Open Access Library Journal. 2:1-9. https://doi.org/10.4236/oalib.1101342

KERN EL, Cobuci JA, Costa CN, McManus CM, Campos GS, Almeida TP. 2015. Genetic association between longevity and linear type traits of holstein cows. Scientia Agricola. 72(3):203-209. https://doi.org/10.1590/0103-9016-2014-0007

MADRID S, Echeverri J. 2014. Association between conformation traits and productive performance in Holstein cows in the department of Antioquia, Colombia. Veterinaria $y$ Zootecnia. 8(1):35-47. ISSN 2011-5415. https://doi.org/10.17151/vetzo.2014.8.1.3

MANAFIAZAR G, Goonewardene L, Miglior F, Crews DH, Basarab JA, Okine E, Wang Z. 2015. Genetic and phenotypic correlations among feed efficiency, production and selected conformation traits in dairy cows. Animal. 10(3):381-389. PMID: 26549643. https://doi.org/10.1017/S1751731115002281

MIGLIOR F, Fleming A, Malchiodi F, Brito LF, Martin P, Baes CF. 2017. A 100-Year Review: Identification and genetic selection of economically important traits in dairy cattle. Journal of Dairy Science. 100(12):10251-10271. https://doi.org/10.3168/jds.2017-12968

MISZTAL I, Legarra A. 2017. Invited review: Efficient computation strategies in genomic selection. Animal. 11(5):731-736. https://doi.org/10.1017/S1751731116002366

JOSÉ MORO MJ, Ruiz LFJ. 1998. Mejoramiento genético de características de conformación en ganado Holstein. Veterinaria México. 29(4):385-398. https://www.medigraphic.com/cgi-bin/new/resumen.cgi?IDARTICULO=15539

PÉREZ-CABAL MA, García C, González-Recio O, Alenda R. 2006. Genetic and phenotypic relationships among locomotion type traits, profit, production, longevity, and fertility in Spanish dairy cows. Journal of Dairy Science. 89(5):1776-1783. PMID: 16606749. https://doi.org/10.3168/jds.S0022-0302(06)72246-9

PICCARDI M, Balzarini M, Bó GA, Funes AC. 2012. Asociación entre las características morfológicas y la producción de leche en vacas Holstein. Revista Veterinaria. 23(2):134137. ISSN: 1668-4834. https://doi.org/10.30972/vet.2321793 
PTAK E, Jagusiak W, Zarnecki A, Otwinowska-Mindur A. 2011. Heritabilities and genetic correlations of lactational and daily somatic cell score with conformation traits in Polish Holstein cattle. Czech Journal of Animal Science. 56(5):205-212. https://doi.org/10.17221/1432-cjas

Ruiz, L.F.J., García, R.A. Cortes H.J.G, 2020. ¿Qué Toro? Evaluación genética cuatrimestral de toros y vacas Holstein para producción, componentes y células somáticas de leche, longevidad y conformación. Estudio No 61, agosto 2020. INIFAP CENID FyMA. Querétaro, México. https://holstein.com.mx/servicios/evaluacionesgeneticas/\#

SAMORÉ AB, Rizzi R, Rossoni A, Bagnato A. 2010. Genetic parameters for functional longevity, type traits, somatic cell scores, milk flow and production in the Italian Brown Swiss. Italian Journal of Animal Science. 9(2):145-152. ISSN: 1828-051X. https://doi.org/10.4081/ijas.2010.e28

SAS Institute. 2019. Statistical Analysis Software SAS/STAT®. Version 9.3, Cary, N.C., USA: SAS Institute Inc., ISBN: 978-1-60764-599-3. https://www.sas.com/es_mx/industry/life-sciences/solution/real-world-evidence.html

TAPKI I, Ziya GÜZEY M. 2013. Genetic and Phenotypic Correlations between Linear Type Traits and Milk Production Yields of Turkish Holstein Dairy Cows. Green Journal of Agricultural Sciences. 3(11):755-761. ISSN: 2276-7770. https://doi.org/10.15580/GJAS.2013.11.072913763

TOLEDO HO, Ruiz-López FJ, Vásquez CG, Berruecos JM, Elzo MA. 2014. Estimation of genetic parameters for milk production in Holstein cattle in Mexico under two modes of production control. Revista Mexicana De Ciencias Pecuarias. 5(4):443-457. ISSN 24486698. https://doi.org/10.22319/rmcp.v5i4.4016

VALENCIA M., Montaldo HH, Ruíz-Lopez FJ. 2008. Parámetros genéticos para características de conformación, habilidad de permanencia y producción de leche en ganado Holstein en México. Técnica Pecuaria en México. 46(3):235-248. ISSN: 00401889. https://doi.org/10.22319/rmcp.v46i3.1807

VAN RADEN PM, Cole J, Parker Gaddis KL. 2021. Net merit as a measure of lifetime profit: 2021 revision. AIPL Research Reports. e-353707. https://www.ars.usda.gov/research/publications/publication/?seqNo115=353707 
WALL E, White IMS, Coffey MP, Brotherstone S. 2005. The relationship between fertility, rump angle, and selected type information in Holstein-Friesian cows. Journal of Dairy Science. 88(4):1521-1528. https://doi.org/10.3168/jds.S0022-0302(05)72821-6

WASANA N, Cho GH, Park SB, Kim SD, Choi JG, Park BH, Do CH. 2015. Genetic relationship of productive life, production and type traits of Korean Holsteins at early lactations. Asian-Australasian Journal of Animal Sciences. 28(9):1259-1265. PMID: 26194223. https://doi.org/10.5713/ajas.15.0034

WAYNE WD. 2017. Bioestadística: Base para el análisis de las ciencias de la salud. Limusa. 4th ed. Pp. 432-470. México. ISBN: 978-968-18-6164-3. https://www.academia.edu/17988752

WELLER JI, Ezra E. 2016. Genetic and phenotypic analysis of daily Israeli Holstein milk, fat, and protein production as determined by a real-time milk analyzer. Journal of Dairy Science. 99(12):9782-9795. https://doi.org/10.3168/jds.2016-11155

World Holstein Friesian Federation. 2018. World Holstein Friesian Federation. Sitio web. http://www.whff.info/index.php

ZAVADILOVÁ L, Šttpková M. 2012. Genetic correlations between longevity and conformation traits in the Czech Holstein population. Czech Journal of Animal Science. 57(3):125-136. https://doi.org/10.17221/5566-cjas 